\title{
The Impact of Nature inspired algorithms on Biomimetic approach in Architectural and Urban design
}

\author{
Natasha Chayaamor-Heil ${ }^{[0000-0002-5582-5988]}$ \\ MAP-Maacc, CNRS-MCC UMR 3495, ENSA PARIS-La-Villette, France \\ natasha.heileparis-lavillette.archi.fr
}

\begin{abstract}
At the time when the value of architecture no longer results from creating shapes in space, but rather fostering relationships within it. A concept of architectural design becomes a strategic process rather than an object. Biological inspiration is dominating the era, and has its impact in diverse domains, including architecture and urbanism. The study of biomimetics bridges the biological functions, processes and organizational principles found in nature with our designs and technologies. Recently there are numerous mathematical algorithms have been developed along with the knowledge transferring process from the life forms to solve the design problems. Output of biomimetics study includes not only physical applications, but also various computation methods that can be applied in different areas. We can learn from biological processes and principles to design and develop a number of different kinds of optimisation algorithms that have been widely used in both theoretical study and practical applications. In this paper, we discuss and present the impact of nature inspired algorithms and digital advanced on biomimetic approach in architectural and urban design. We demonstrate how architects reuse bio-inspired computing to solve complex problem or optimise their designs, and nonetheless, how architects use algorithmic architecture software to directly transpose the complexity of nature's principles into their design process.
\end{abstract}

Keywords: Biomimetics, Biological process, Nature inspired algorithms, Architectural and Urban design.

\section{Introduction}

Nature has always been an inspirational source for architects and designers. This inspiration has given highly efficient designs in various fields. One of the best concept in which, we see nature as the influential source of inspiration. Biomimetics means imitating of biological processes. Biomimetics is a new science and design discipline that studies nature's models and then emulates these forms, processes, systems and strategies to solve the problems of our time [1]. In September 2015, the Economic, Social and Environmental Council of Paris issued a noticed entitled 'Biomimicry: taking inspiration from nature to innovate sustainably' [2]. Architecture is presented as one of the promising areas of application and there is evidence of an emerging of biomimetics in architectural design activity capable of responding to current envi- 
ronmental issues. Undoubtedly, architects have been inspired by nature to stimulate a novelty in their designs, but beyond the appearance of a tree, a branch or a simple leaf, there are mathematical patterns at play in nature. Understanding nature's basic algorithms allows architects and designers to explore natural systems that can be translated into efficient designs and awe-inspiring architecture. In fact, nature as inspiration is often combined with mathematics in order to move beyond the superficial inspiration and realize a design [3]. The fluid order in nature is characterized by repetition, growth and change with the fundamental rules that generate order in nature not being absolute but relative, flexible and soft [4]. In contemporary architecture design, digital media is increasingly being used not as a representational tool for visualization but as a generative tool for the derivation of form and its transformation [5]. The use of computational techniques increasingly saturates development biology, from the acquisition, processing and analysis of experimental data to the construction of models of organisms. Similar to the biological processes, it is a process of shape/pattern development enabled by computation but in digital environment. However when it comes to algorithms it is not just about software's and computers, they describe a process of logical thinking [6]. Yet beyond this, a computational approach to architecture enables the generation of the previously unseen forms that can longer be conceived of through traditional methods become possible, thus opening up new realms.

\section{The overview of Biomimetics}

\subsection{The emergence of Biomimetics}

In the 1950s biophysicist and engineer Otto H. Schmitt established the field of biomedical engineering and proposed the term 'biomimetics' for this new science of the emerging engineer. The term 'bionics' in the United States, invented by the neurologist Jack E. Steel, and 'bionik' in Germany, originating from the work of the biologist Werner Nachtigall, appeared in the late 1950s. These terms referred to the study of the structures and functions of biological systems as models for the design and engineering of materials and machines. This field of investigation developed during the 1960 s and remained mainly for robotics. During the 1970s, the concept of bionics became widespread among engineers and designers within companies. Nachtigall describes the concept as: 'Bionics is the process of taking nature as a source of inspiration for independent technical design' [7]. The term 'biomimicry' appeared in 1980 and was popularized by the biologist and environmentalist Janine Benyus, author of the book Biomimicry: Innovation Inspired by Nature [8]. Biomimicry is defined in her book as a new science that studies nature in order to imitate it or to draw inspiration from it to solve human problems. The concept of biomimicry, as supported by J. Benyus, proposes to draw inspiration from the brilliant ideas developed in nature to design our innovations from a perspective of sustainability. Benyus suggests looking at nature as a model, measure or mentor. 


\subsection{The process sequences in biomimetic research}

If we consider the biomimetic design process as a whole, from the initial idea to the final product, two approaches have been identified [9]. The first approach starts from a human need or a design problem then examines the ways in which organisms or ecosystems found in nature solve this problem. This is a problem-oriented approach (Top-down or design looking to biology). This approach is actually carried out by designers who, after identifying initial objectives and design parameters, seek solutions in the nature world.

The second approach is to identify a particular characteristic, behavior, or function in an organism or ecosystem, and then look for the design problem that could be addressed. It is a solution-oriented approach (Bottom-up or biology influencing design). This approach is where knowledge in biology influences human design. It is conducted by biologists or scientists who are looking for possible applications relevant to the design.

\subsection{The levels of biomimetics}

Biomimetics inspires architecture in different levels as biology does in the nature and these levels can be summarized under three categories: form, process and ecosystem. Form and processes can mimic in an ecosystem. This approach is methodized by Zari [10] to specifically apply to a design or an architectural problem. The three levels mentioned above are rearranged and separated into five sub-levels (See figure 2) and explained how biomimetics is considered for a design problem.

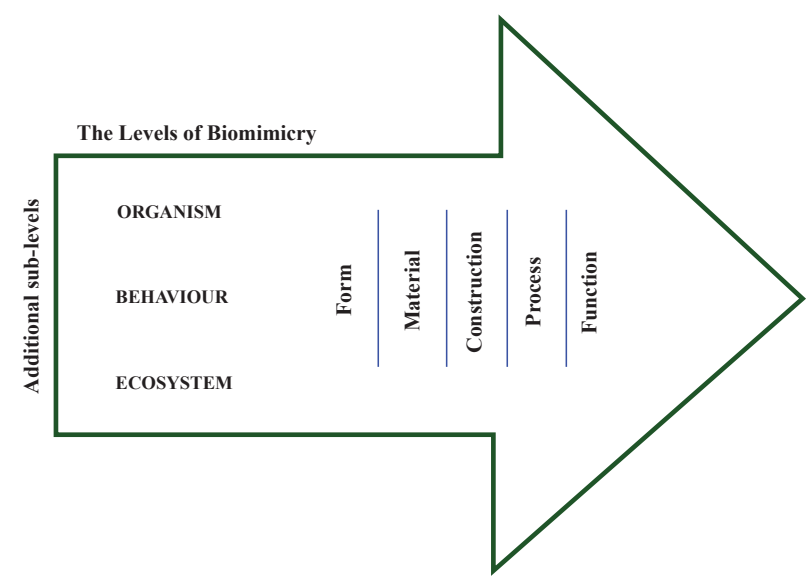

Fig. 1. Theoretical framework for the levels and additional sub-levels of biomimetics in architecture, adapted from [10]

The organism level refers to a specific organism like a plant or an animal and may involve mimicking part of or the whole organism, solutions related to efficient energy usage and materials, which are established already. In brief, it is mimicking an organism's physical attributes. 
The behavior level refers to mimicking organism's behavior, to explore and understand how an organism relates and behaves to a larger context. It is possible to understand this level with observing how an organism tends to operate in its environmental capacity and within limits of energy and material availability.

The ecosystem level of biomimetics intends to create a whole ecosystem, which incorporates the other two levels to achieve a sustainable environment. This means a deep comprehension of ecology and the regenerative process of the nature, which may begin from a small scale and tends to lead to a bigger scale of thinking like green cities or eco-cities.

Within each of the three levels, a further five possible dimensions to the mimicry exist. The design may be biomimetic for example in terms of what it looks like (form), what it is made out of (material), how it is made (construction), how it works (process) or what it is able to do (function) [10].

\section{$3 \quad$ Algorithms in nature}

The real beauty of nature inspired algorithms lies in the fact that it receives its sole inspiration from nature. They have the ability to describe and resolve complex relationships from intrinsically very simple initial conditions and rules with little or no knowledge of the search space. Nature is the perfect example for optimization, because if we closely examine each and every features or phenomenon in nature it always find the optimal strategy, still addressing complex interaction among organisms ranging from microorganism to fully fledged human beings, balancing the ecosystem, maintaining diversity, adaptation, physical phenomenon like river formation, forest fire, cloud, rain etc. [12]. Nature inspired algorithms is meta-heuristics that mimics the nature for solving optimization problems opening a new era in computation [11]. For the past decades, numerous research efforts have been concentrated in this particular area. Still being young and the results being very amazing, broadens the scope and viability of Bio Inspired Algorithms (BIAs) exploring new areas of application and more opportunities in computing.

Optimization is a commonly encountered mathematical problem in architectural and engineering disciplines. It literally means finding the best possible/desirable solution. Optimization problems are wide ranging and numerous, hence methods for solving these problems ought to be, an active research topic. Optimization algorithms can be either deterministic or stochastic in nature. Former methods to solve optimization problems require enormous computational efforts, which tend to fail as the problem size increases. This is the motivation for employing bio-inspired stochastic optimization algorithms as computationally efficient alternatives to deterministic approach. Meta-heuristics are based on the iterative improvement of either a population of solutions (as in Evolutionary algorithms, Swarm based algorithms) or a single solution (eg. Tabu Search) and mostly employ randomization and local search to solve a given optimization problem. 


\subsection{A multidisciplinary approach}

Interdisciplinary collaboration is necessarily essential in biomimetic design process where the knowledge from different fields converges, which leads to innovation and increase the novelty of design ideas. Nature is providing a tremendous amount of information implemented into these scientific fields, assisting contemporary investigations that vary from the analysis of the human body's structural element: bone's micro-mechanical configuration [13], to the effect of the micro-fibril orientation into plant growth. However it must also be understood that as these design processes requires the collaboration between the architect and the biologist, a similar collaboration might be required between an architect and a computer programmer or mathematician to create the complex algorithms that are used in nature. As the architect though can create the concept and workflow may not be skilled enough in making the algorithm. In nature, the development of the life form and structure is complex; it is a kind of free-from, free-edge and non-linear structure, which can sometimes be modeled mathematically. There is a mathematics lies behind numerical pattern in nature, thus it is not a simple task for an architect to understand and demonstrate more advanced programming techniques with processing that focus on algorithms and simulation of natural formations. There are some distinct collaboration between computer programmers and architects for an advanced research in architectural project that produce a free-from and complex structure as in nature. One known is the work of Prof. Helmut Pottmann, who has collaborated with a number of architects (an example of a project, figure 2) [15]. He is also the author of a notable book 'Architectural Geometry' [14]. The book demonstrates some cutting-edge of complex geometrical modeling that needs a mathematically well trained to be able to produces such a models. These include central concepts on freeform curves and surfaces, differential geometry, kinematic geometry, mesh processing, digital reconstruction, and optimization of shapes [14]. Likewise, these concepts appear in nature.
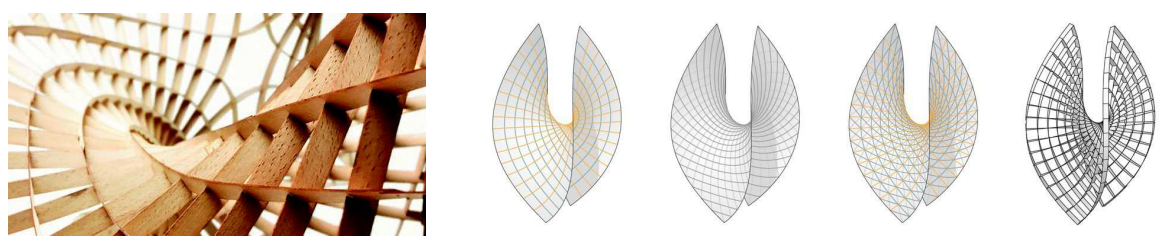

Fig. 2. Project Designing Grid Structures Using Asymptotic Curve Networks, Humanizing Digital Reality: Design Modeling Symposium Paris 2017 [15]

Today, there are few known examples of architectural construction that are completely generated from scratch by algorithms based on biomimetic approach, for example, The algorithm developed by Chris Bosse for the design of the National Aquatics Centre in Beijing, the 'Water cube' [16], the building's form is inspired by natural formation of soap bubbles, crystals, cells and molecular structures, these are the most efficient subdivision of 3 dimensional space with equally sized cells. In this design, it moves the algorithmic process one step further where a single material system produces structure and at the same time defines space. Structural stability is a priori as- 
sured by the design choice itself, the formation of a stable configuration of the geometry of bubble packing that also occurs spontaneously in many natural systems.
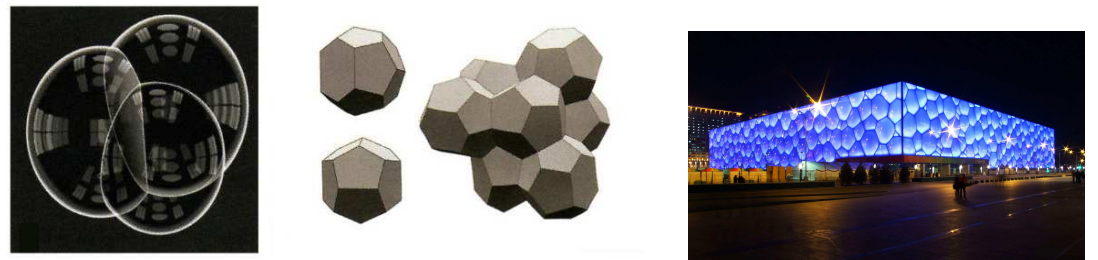

Fig. 3. (Left) The efficiency of soap-bubble structures is exploited with Weaire-Phelan geometrical principle, (Right) National Aquatics Centre in Beijing, the Watercube by Chris Bosse, 2008, [17]

The next chapter, we will present some case studies on the utilization of nature inspired algorithms within a framework of biomimetic design process in architecture along with the use of computational design and the relevant applications in architectural and urban domain.

\section{The biomimetic framework in architecture}

Biomimetic architecture is a contemporary approach of architecture that seeks innovative and sustainable solutions in nature [18], without attempting to replicate its forms, but by seeking to understand the rules that govern them. The objective of biomimetic architecture no longer consists solely in giving form and measurement to space but also in developing synergistic relationships between the construct and its environment. The heuristic approach to biomimetics consists in bringing architecture to " $v i$ talism' beyond the mechanistic view of life [19]. The biomimetic architecture could be at the origin of a transformation of the role of architects evolving from the control of the nature to a lasting participation with the nature.

Original method of biomimetic architecture is a cross-disciplinary approach between biology and architecture. This method is initially called 'Bau-Bionik', coined by an architect, Göran Pohl and a biologist, Werner Nachtigall [20]. As a result of a combined effort by the two disciplines describes the principles which can be used to compare nature, design and technology, how biology can be used as a source of inspiration and 'translated' in building and architectural solutions along with current advanced technology. However, it is not a trivial task to understand the principles that govern the living, especially for architects who need to search for an elegant biological analogy and transfer it to solve problem in architectural designs. One must only then be cautious of too direct interpretation [20]. Inspirations from nature for architecture will not function if they do not well abstract within the context of an interdisciplinary analogue.

In the book 'Bionik als Wissenschaft' [21], which applies the theory of cognition to biomimetics, signified this process in three-step: Research $\rightarrow$ Abstraction $\rightarrow$ Implementation. By observing a cognitive biomimetic design process within the context of 
an interdisciplinary, 'identification' and 'abstraction' often proves to be one of the most important as well as most difficult steps in a biomimetic project [9]. Thus, we have found two common difficulties for an architect to apply biomimetic methodology into their design process, we define the difficulties in two transitions; 1 . What to look for in nature? 2. How to interpret natures' principles and transfer into design phase?

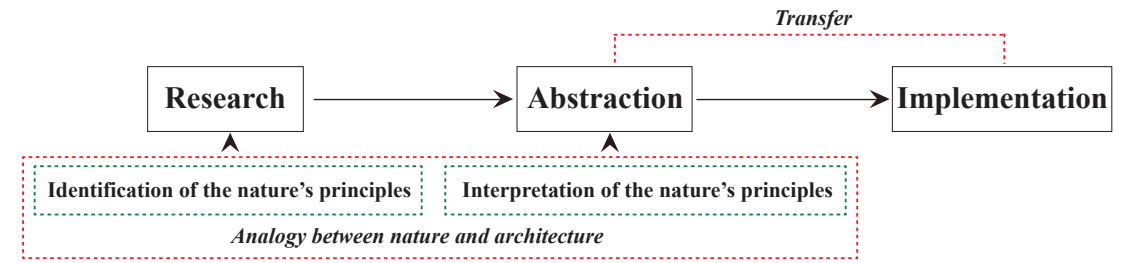

Fig. 4. The two transitions of a cognitive biomimetic design process within the context of an interdisciplinary, adapt from [21]

In view of these two transitions, it is possible to distinguish two postures of the use of biomimetic approach in architecture: an indirect posture where the architects explore biologically inspired computing as a tool derived from biomimetic interdisciplinary collaboration between biologists and computer scientists, and a direct posture where the architect integrates directly an interdisciplinary biomimetic activity and use computational algorithms to transpose biological knowledge in its architectural design process.

\subsection{An indirect biomimetic methodology in architectural design activity}

In many bio-inspired architectural design activity, architects construct design methods but the form and technical processes of their projects can be completely different from what is found in nature. These design methods generally rely on computer techniques such as particle systems, genetic algorithms or multi-agent systems whose functioning is bio-inspired. Bio-inspired computing, short for biologically inspired computing, is a field of research that breaks down into subfields including connectionism, social behavior and emergence. It is close to artificial intelligence or artificial life. It is related to the fields of biology, computer science and mathematics. Briefly, it is a matter of creating innovative algorithms to solve known problems, such as optimization problems, for example, based on phenomena observed in nature. The following case study presents the use of a biomimetic algorithm capable of designing an optimized transport network between different cities while taking into account a certain number of characteristics (population, points of interest, possible breakdowns on the network, etc.).

\section{Case study: Biomimetic Algorithms: Slime mold inspired network design}

It all began in the early 2000s when a team of Japanese and Hungarian researchers discovered that a kind of slime mold called Physarum Polycephal is able to find its way in a labyrinth [22]. This mold appears to be intelligent and capable of exploring 
its environment to find the shortest paths to food sources [23]. Based on these observations, an algorithm was constructed; mimicking the behavior of Physarum Polycephal called the Physarum Solver [24]. This algorithm is able to find an optimal path in a network and proves to be more efficient than the classical algorithms when the number of nodes of the network is very important. This stunning algorithm can for example solve problems of optimization of a transport network. How to connect cities in a minimum of lines with maximum efficiency, that is to say taking into account population density, geography, possible breakdowns or accidents that may occur on the network and etc. It has been tested in several cities and countries: the New York City street network, the railway network in Japan and other transportation networks in Mexico and China [25]. It is clear that this type of algorithm can be used by urban planners or architects to design, for example, optimized and resilient distribution networks (for example, energy, water and etc.).
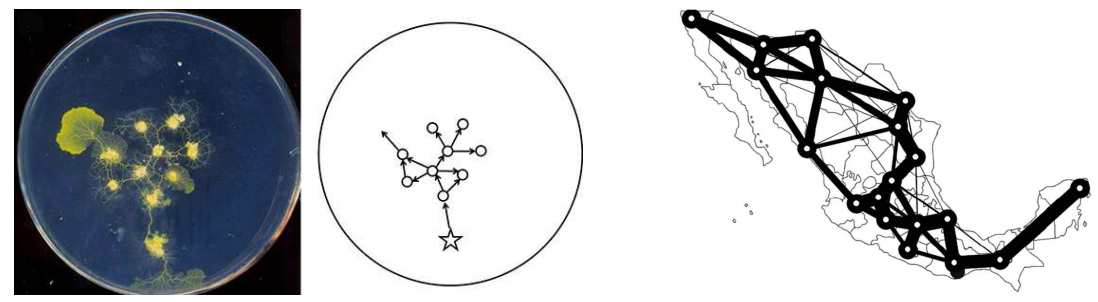

Fig. 5. (Left) The behavior of the Physarum Polycephal observed in a petri dish and the bioinspired mathematical model of the exploratory behavior of Physarum Polycephal [26], (Right) the transportation network in Mexico City developed by the Physarum Solver [25]

The following figure describes a possible architectural design process resulting from collaboration between biologists, computer scientists and architects.

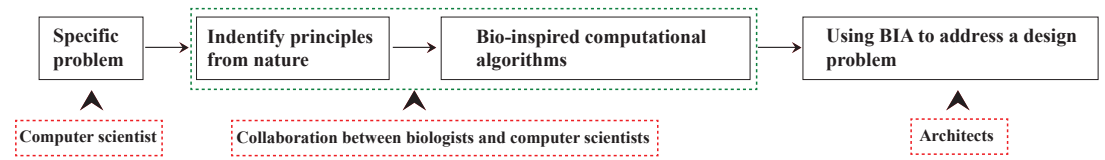

Fig. 6. The steps of an indirect biomimetic design process in architectural activity, [27]

This framework of an indirect biomimetic methodology does not directly concern the architectural field. It produces innovation (optimization) in the field of computing. It is clear that in such a process, it is the algorithm used that is innovative because it is designed in a biomimetic approach between computer scientists and biologists. The architect, by reusing these algorithms, produces an optimized architectural and urban design but not necessarily innovative. That's why we called this process an indirect biomimetic methodology.

\subsection{A direct biomimetic methodology in architectural design activity}

In the case study presented below and unlike the previous one, there is direct collabo- 
ration between the architect and the biologist and the use of computational algorithms to support the transfer of nature's principles into the design process. The collaborative design activity consists of the initial phase 'specific design problem' that architect specify, then the architects themselves observe in nature and collaborate with biologists to identify the strategy and abstract the design principles from nature to solve the problem in architectural and urban design.

\section{Case study: District 11 - Skolkovo Innovation center and Thermal regulation system of Emperor penguins, 2017}

Inspired by the complex social behaviour of group organization of emperor penguins that protects from the cold and save energy. Agence d'Architecture A. Bechu \& Associés collaborates with biologists to study the thermal regulation system of emperor penguins on how a penguin colony works as a system to allow all penguins a chance to be warm. The architects use the computational algorithm to transpose this principle into a complex town planning, which will be housing researchers and their families in single-family homes as well as providing them with a living environment that encourages social interaction. Much like penguins on an ice shelf forming a circle to share their heat, a hundred villas are grouped ten by ten in a vast clearing surrounded by a waterway able to drain away the melting snow. Each of the villas will be unique, giving occupants their own identity within the urban ensemble, but will all feature a modular concrete frame, green roof, and use of renewable energy and water recycling. Public functions and common shared services will be located in a central area, in order to create a social link between residents [28].
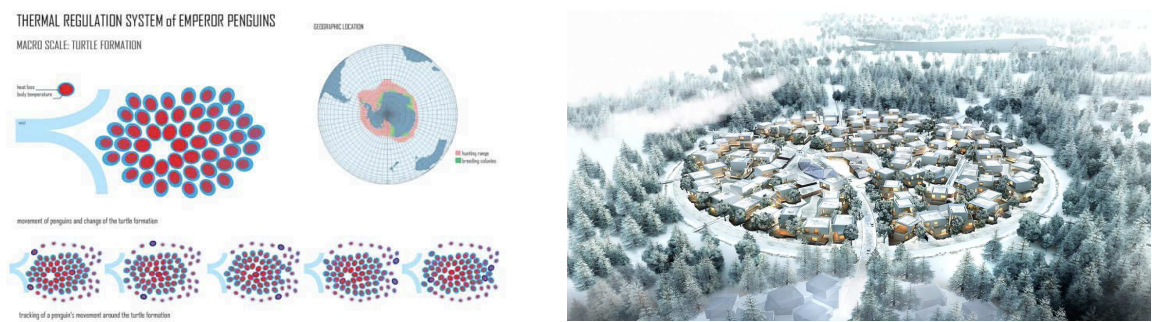

Fig. 7. (Left) Thermal regulation system of Emperor penguins [29], (Right) District 11, Skolkovo innovation center, Russian [28]

Case study: AEOMIUM: a fog sensor dome to produce drinking water in arid area. Site study: Burkina Faso, Africa, 2017

This architectural project 'AEONIUM' [30] proposes a fog sensor built near a typical rural village of Burkina Fuso. The operation of the dome is inspired by the metabolism of Xerophile plants (for example, succulents) and their different strategies developed to capture and store the water contained in the ambient air. By bringing the source of drinking water to the villages, the installation is a tribute to the tradition of the water carriers since it facilitates the activity while creating a pleasant meeting 
place. The project is realized by the groups of students within the program of Biomimetic project applied algorithmic and digital method for architectural design and fabrication at the Laval University in Canada [31]. The project was entirely realized with the help of an algorithmic software Grasshopper [32] and algorithmic families (fractals, AC, AG, complexity, etc.) while adopting a biomimetic approach. The architects have made an analogy between the principles of the plant and the design concept and once the biological principles are understood as a design task, the architect transcript them in Grasshopper. This new way of reasoning allows the architects to better understand the functioning of the biological models studied, since they respond to an algorithmic logic like Grasshopper. In this project, the algorithms in grasshopper first and foremost helped the architects to develop all the kinematics of opening and closing of their protective envelope, the principle of which stemmed from the in-depth study of the functioning of stomata in Xerophile plants.

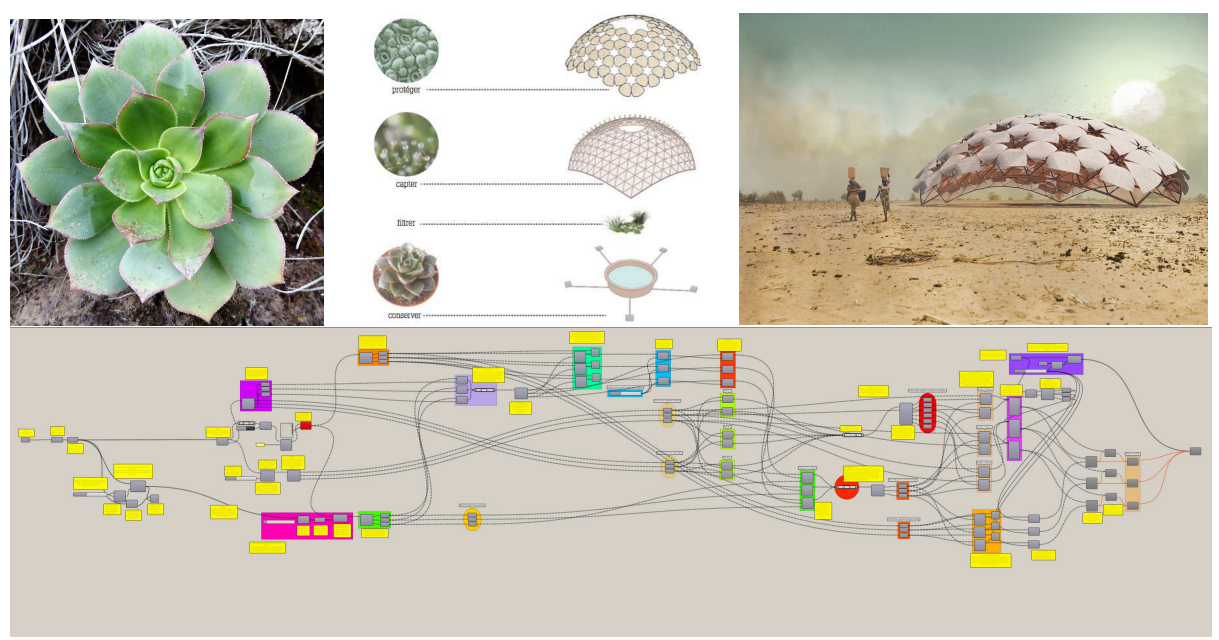

Fig. 8. (From top left to right) The succulent plant Aeonium percarneum [33], the functionality of the fog sensor dome, the rendering of the dome prototype [30], (Below) The Grasshopper canvas of the AEONIUM design process [30]

From the two case studies above, the architect seeks to understand application of algorithm based on biomimetic principles to the architectural context. The architects involve directly in biomimetic activity, from the initial to the final phase. Additionally, at present, the algorithm scripting in Grasshopper tool [32] are being used to transpose nature's principles towards biomimetic approach within the architectural domain. Grasshopper is an extremely powerful tool but unfortunately it's still little known specially in bio-inspired architectural field. In somehow, any natural principle can be retransmitted as lines of code to determine a sequence of actions composing the biological system, but this way requires strong knowledge in computer science and code, which is not necessarily the case in an architect. With Grasshopper it is now possible to simplify all this procedure through clusters. Indeed thanks to these small 'boxes' closing actions predefined by the software, it is possible to design sequences 
of operations so as to reproduce the biological system explicitly and visualize the result in real time.

The following figure describes a possible architectural design process resulting from direct collaboration between architects and biologists and the use of computational algorithms to transpose nature's principles into design phase.

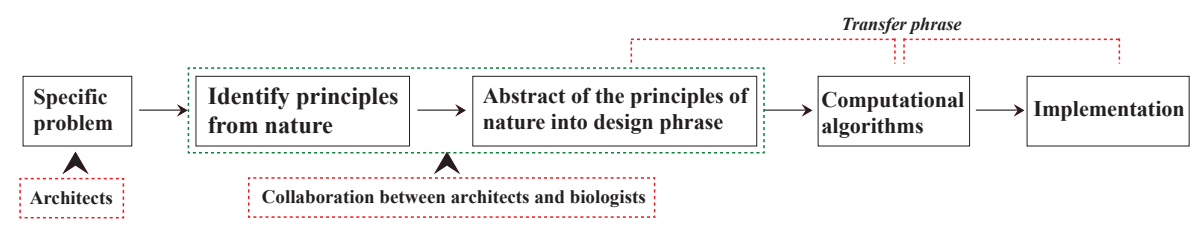

Fig. 9. The steps of a direct biomimetic design process in architectural activity, [27]

The unique framework of biomimetic design is directly related to the architectural field. It produces innovation directly in the field of architecture. This is a direct biomimetic design process because there is an effective interdisciplinary collaboration between architects, biologists and computer programmer, the latter welcoming with great interest the idea of developing this type of collaboration.

\section{Conclusion}

This paper is an investigation into the development of design method based on biological principles that are applied and correlated with nature inspired algorithm and computational design in the field of architecture. It is important to materialize the architectural output based on application of biomimetics. It can be concluded that the stated methodological frameworks enable the designer to produce an efficient architecture in terms of innovation and sustainability. But at the same time this approach relies on very specific knowledge, skills and tools. Architects have been searching for answers from nature to their complex questions about different kinds of structures, and they have mimicked a lot of forms from nature to create better and more efficient structures for different architectural purposes. Without computers these complex ways and forms of structures couldn't been mimicked and thus using computers had risen the way of mimicking and taking inspiration from nature because it is considered a very sophisticated and accurate tool for simulation and computing, as a result designers can imitate different nature's models in spite of its complexity. Bio-inspired algorithms are going to be a new revolution in computer science. Nevertheless, natureinspired algorithms are among the most powerful algorithms for optimization, which is going to have a wide impact on future generation computing. Though these algorithms are becoming widespread in many design and fabrication industries, perhaps their best use is in architectural design, where they can enable architects to work in intuitive and nondeterministic ways. Thus new and innovative designs can be produced that achieve structural and environmental performances that were once considered to be post-design optimization processes. 


\section{Acknowledgements}

This article is part of an extended research of Biomimicry in Architecture: State, methods and tools, which is published in les Cahiers la recherche architecturale, urbaine et paysagère, issue Innover 2018 (https://journals.openedition.org/craup/309). I would like to thank François Guéna, the director of Map-maacc UMR 3495, Paris, France and Pierre Côté, Professor at Laval University, Canada who provided expertise that greatly assisted on this subject.

\section{References}

1. Biomimicry Guild, Innovation Inspired by Nature Work Book, Biomimicry Guild, (2007).

2. Ricard, P., Le biomimétisme : s'inspirer de la nature pour innover durablement, Les projet d'avis du Conseil économique, social et environnemental (CESE), Paris, septembre (2015).

3. Toyo, I., Algorithms are Nothing More than an Opportunity to Create Architecture that Respires, pp. 36-45, The Japan Architect, Japan (2010).

4. RianIasefMd, Tree - Inspired Dendriforms and Fractal like Branching Structures in Architecture: A Brief Historical Overview, Frontiers of Architectural Research, vol. 03, Issue no.3, pp. 298-323, ELSEVIER, (2014).

5. Kolarevic B., Architecture in the Digital Age - Design \& Manufacturing, Spon Press Taylor \&Fransic Group, New York, London, (2003).

6. Bagul, P., Uke, N., Algorithms in Architectural Design, International Journal of Electronics Communication and Computer Engineering, Volume 6, Issue (4) July, India (2014)

7. Wahl, D. C.,Bionics vs. biomimicry: from control of nature, WIT Transactions on Ecology and the Environment, 87, 289-298, W. press, Éd., (2006).

8. Benyus, J. Biomimicry: Innovation Inspired by Nature, New York, Harper Collins Publishers, (1997).

9. Speck, T., Process sequences in biomimetic research, Design and Nature IV, Brebbia (éd.), pp. 3-11, WIT Press (2008).

10. Zari, M.P., Biomimetic Approaches to Architectural Design for Increased Sustainability, Sustainable Building Conference, Auckland, (2007).

11. Siva Sathya, B., A Survey of Bio-inspired optimization Algorithms, International Journal of Soft Computing and Engineering, Volume2, Issue(2), (2012).

12. Sidhu, T., Samarabandu, J., Premaratne, U., A New Biologically Inspired Optimization Algorithm, Fourth International Conference on Industrial and Information Systems, ICIIS, pp. 28-31, Sri Lanka (2009).

13. Huiskes, R., If bone is the answer, then what is the question, Journal of Anatomy 197, pp. 145-156, Netherlands, (2000).

14. Pottmann, H., Asperl, A., Hofer, M. and Kilian, A., Architectural Geometry, Bentley Institute Press (2007).

15. Shiling, E., Hitrec, D., Barthel, R., Designing Grid Structures Using Asymptotic Curve Networks, Humanizing Digital Reality: Design Modeling Symposium Paris 2017, Springer, (2018).

16. Water cube Homepage, http://www.chrisbosse.de/watercube/, last accessed 2018/05/29.

17. https://en.wikipedia.org/wiki/Beijing_National_Aquatics_Center, last accessed 2018/05/29

18. Pawlyn, M., Biomimicry in Architecture, London, RIBA publishing, (2011).

19. Gruber, P., The signs of life in architecture, Bioinspiration \& Biomimetics, (2008). 
20. Nachtigall, W., Bau-Bionik: Natur $\leftarrow$ Analogien $\rightarrow$ Technik, Berlin, Springer/Auflage, (2003).

21. Nachtigall, W., Bionik als Wissenschaft: Erkennen-Abstrahieren-Umsetzen, Berlin, Springer/Auflage, (2010).

22. Nakagaki, T. et al., Intelligence: Maze-solving by amoeboid organism, Nature, 407, p. 470, (2000).

23. Nakagaki, T. et al., Otaining multiple separate food sources: behavioural intelligence in the Physarum plamodium, The Royal Society, 271, pp. 2305-2310, (2004).

24. Tero, A. et al., Physarum solver: a biologically inspired method of road-network navigation, Elsevier Science, (2005)

25. Mahadevan, S., A Biologically Inspired Network Design Model, Nature research journal, Scientific Reports 5, (2015).

26. Adamatzky, A., Slime mould processors, logic gates and sensors, The Royal Society Publising, Philosophical Transactions of The Royal Society a mathematical, physical and engineer science, vol. 373, $n^{\circ} 2046,(2005)$.

27. Chayaamor-Heil, N., Guéna, F., Hannachi-Belkadi, N., Biomimicry in Architecture: State, methods and tools, Les Cahiers de la recherché Architecturale Urbaine et Paysagère, http://journals.openedition.org/craup/, (2017).

28. Agence d'architecture A.Bechu Homepage, http://www.anthonybechu.com/fr, last accessed 2018/02/1.

29. Asknature Homepage, https://asknature.org, last accessed 2018/03/15.

30. AEONIUM Homepage, https://aeonium.wixsite.com/project, last accessed 2018/05/29.

31. AENIUM project is part of the program Méthodes en Architecture en Fabrication numériques ARC-6046, Automne 2017, University Laval, by Prof. Pierre Côté, Marie-Jeanne Allaire-Côté, Théo Jarrand, Marc-Antoine Juneau and Josianne Ouellet-Daudelin.

32. Grasshopper: Algorithmic modeling for Rhino Homepage, http://www.grasshopper3d.com, last accessed 2018/01/15.

33. Wikipedia Aeonium, https://fr.wikipedia.org/wiki/Aeonium, last accessed 2018/05/29. 